\title{
辨体 - 辨病 - 辨证诊疗模式在 2 型糖尿病患者中的应用
}

\section{Application of Treatment Model by Differentiation of Physique, Disease and Syndrome in Patients}

\section{with Type 2 Diabetes}

\section{蒋茂剑 薛金志}

Maojian Jiang Jinzhi Xue

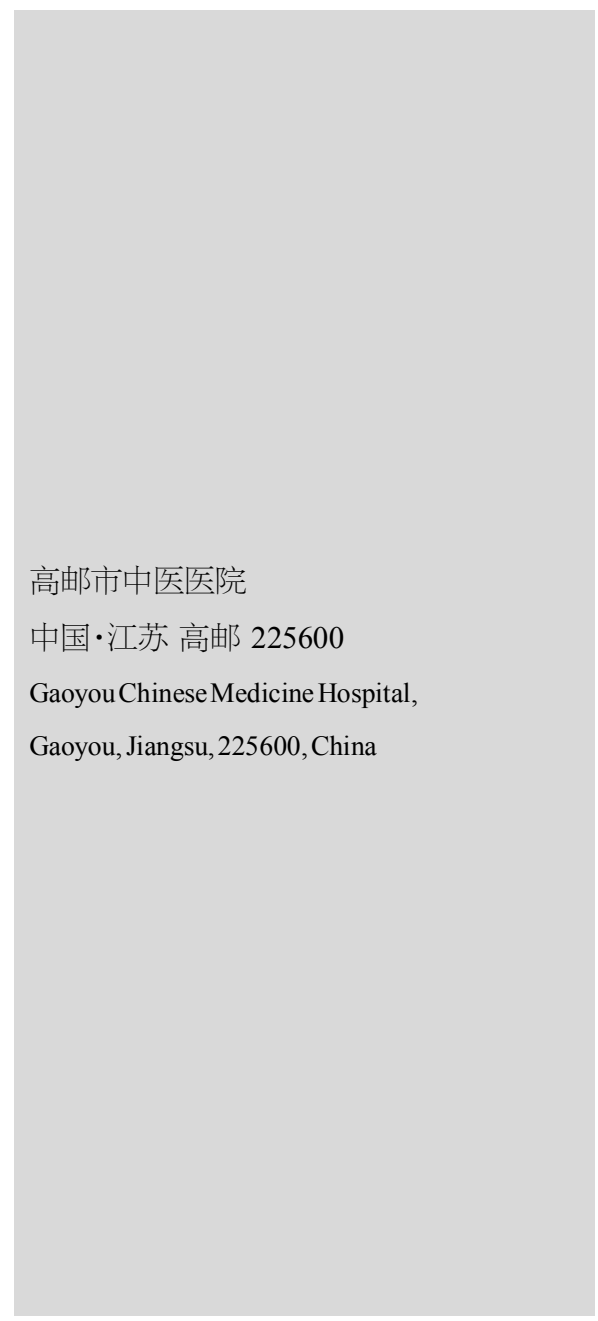

【摘 要】目的: 探究对于 2 型糖尿病患者的治疗, 应用辨体 - 辨病 - 辨证诊疗模式对其进 行辨证诊治的临床价值。方法: 论文的研究对象是笔者工作单位的内分泌科于 2019 年曾收 治的患者,将评估为 2 型糖尿病的 126 名患者进行纵向比较分组。将前六个月接受常规上、 中、下消辩证诊疗模式的 63 例患者视为对照组; 将后六个月, 接受辨体 - 辩病 - 辨证诊疗模 式的 63 例患者作为观察组, 观察两组患者的康复效果。结果: 接受辨体 - 辨病 - 辨证诊疗模 式的观察组患者无论在空腹血糖水平、OGTT 结果的改善上都明显优于对照组患者。结论: 应用辨体 - 辩病 - 辨证诊疗模式, 从中医角度讲, 可以明确辩证分型、大幅改善患者的消渴 症状。从西医角度讲,应用此治疗模式可以改善胰岛功能,并有利于血糖的控制。

【Abstract】Objective: To explore the clinical value of treatment model by differentiation of physique, disease and syndrome in patients with type 2 diabetes. Methods: A longitudinal comparison was conducted on 126 patients who were admitted in endocrinology department of author's workplace with type 2 diabetes in 2019. The 63 patients who received common treatment model in the first 6 months were treated as the control group while the 63 patients who received the treatment model by differentiation of physique, disease and syndrome in the last 6 months were taken as the observation group, and the rehabilitation effect of the two groups was observed. Results: The patients in the observation group were significantly better than those in the control group in terms of fasting glucose level and OGTT results. Conclusion: From the perspective of Traditional Chinese medicine, the application of the treatment model by differentiation of physique, disease and syndrome can clearly differentiate dialectically and greatly improve patients' thirst elimination symptoms. From the western medicine point of view, the application of this treatment model can improve the function of islet and is beneficial to the control of blood sugar.

【关键词】辨体 - 辨病 - 辩证; 2 型糖尿病

【Keywords 】differentiation of physique; disease and syndrome; type 2 diabetes

【DOI】10.36012/pmr.v2i2.1800

\section{1 引言}

在 21 世纪初, WHO 就曾预测糖尿病的发病率将于中国 等发展中国家大幅提高。随着中国物质水平的提高, 2 型糖尿 病的发病率也随之激增 ${ }^{[1]}$ 。因此,如何改善 2 型糖尿病患者的 治疗效果就成为值得研究的课题。笔者根据三十余载临床实 践经验中发现, 对于 2 型糖尿病患者的治疗, 应用王琦院士国
医大师创立的辨体-辨病-辨证诊疗模式有利于大幅改善患者 的消渴症状, 提高预后效果, 现详细介绍体会如下。

\section{2 资料与方法}

\section{1 一般资料}

共有 126 例患者被选为本次辨体-辨病-辨证诊疗模式实 验观察对象,均为 2019 年就诊于笔者工作单位的内分泌科患 
医学理论研究 Medical Theory Research

者, 主要为医院内分泌科门诊、病房住院患者。通过分析 126 例患者的临床表现,综合评估判断各项指征,均符合 2 型糖尿 病沴断标准。

\section{2 方法}

将 2019 年笔者工作单位收治评估为 2 型糖尿病的 126 名患者进行纵向比较分组。将前六个月的 63 例患者视为对照 组, 接受以上、中、下消的常规辨证诊疗模式, 将后六个月收治 的 63 例患者作为观察组, 应用辨体-辨病-辨证诊疗模式, 观 察两组患者的康复效果。

\section{3 结果}

两组患者的基本情况对比如表 1 所示。

表 1 两组患者的基本情况对比 (OGTT2 小时血糖)

\begin{tabular}{c|c|c|c|c}
\hline 组别 & 人数(例) & & 空腹血糖 $(\mathrm{mmol} / \mathrm{L})$ & $\operatorname{OGTT}(\mathrm{mmol} / \mathrm{L})$ \\
\hline \multirow{2}{*}{ 对照组 } & \multirow{2}{*}{63} & 治疗前 & $8.7 \pm 1.4$ & $12.8 \pm 1.7$ \\
\cline { 3 - 5 } & & 治疗后 & $6.6 \pm 0.7$ & $8.7 \pm 1.4$ \\
\hline \multirow{2}{*}{ 观察组 } & \multirow{2}{*}{63} & 治疗前 & $8.9 \pm 1.6$ & $12.6 \pm 0.9$ \\
\cline { 3 - 5 } & & 治疗后 & $5.2 \pm 0.4$ & $7.4 \pm 1.1$ \\
\hline
\end{tabular}

\section{4 讨论}

\section{1 辨体}

清楚地辨别患者的体质成为后续开展辨证及治疗的基 础, 而在对于 2 型糖尿病患者体质辨别的作用, 主要表现在以 下两个方面:第一, 解决了 “无证可辨”的问题。对于一些糖尿 病临床症状不明显, 如 IFG、IGT 等除血糖升高外无任何症状 的患者, 医师对其辨证时就较为困难, 这时对患者体质的辨 别就可能成为对于疾病诊断的唯一辨证线索。第二, 清楚患 者的体质有利于正确用药。通过中医手段干预 2 型糖尿病的 特点是个性化较强、一人一方。例如, 对于肥胖且嗜酒的患 者, 可判断其体质为水饮内停且湿浊内蕴, 用药应当不单单重 视糖尿病患者普遍阴虚的病机，更应根据患者的体质用以健 脾利湿化浊之力较强的药物 ${ }^{[2]}$ 。

\section{2 辨病}

现代临床医学认为,糖尿病是一种代谢疾病群, 其主要特 征为血糖升高 ${ }^{[3]}$ 。中医学认为, 糖尿病包含于消渴范围内, 但在 本次实验中, 有一定比例的 2 型糖尿病患者无任何消渴症状。 因此，笔者首先对这些症状不明显的患者运用现代医学检查 手段,评估其胰岛 $\beta$ 细胞功能, 对有可能存在的并发症进行筛 查, 若单纯对这些患者以消渴症论治, 则可能误诊或漏诊。对 于已经发生酸中毒或高渗性昏迷等急性并发症的糖尿病患者 而言, 更应及时诊断与干预, 为其实施胰岛素静注、调节水与
电解质紊乱等抢救措施。因此, 对于 2 型糖尿病患者, 应当首 先利用现代医技手段明确诊断, 再利用中医四诊合参, 掌握其 内在规律与病因病机, 根据其消渴症的具体分型进行相应的 论治方式, 这就是王琦院士所阐述的观点“先辨病, 再辨证”, 以取中西医相互之长,补各自之短。

\section{3 辨证}

在上述辨病的基础上,调整患者代谢与生理功能的紊乱。

证, 是机体在疾病发展过程中某一阶段的病理概括。对于 2 型糖尿病患者的辨证, 应当以其病史、体征与各项检查结果 等临床资料为基础, 运用中医辨证思维对其证型进行分析 ${ }^{[4]}$ 。 应当分析并总结患者的病因病机、病位病性以及邪正对比等 情况, 应当以王琦院士的观点为基础: 病中辨证, 以病统证, 将 辨病与辨证相结合。传统中医临床中对于糖尿病的辨证, 往往 通过上、中、下消的方式进行, 但笔者认为传统的三消辨证法 已经不再适应现代临床的需要, 随着王琦院士辨体-辨病-辨 证诊疗概念的提出, 这种诊疗模式在临床中逐渐被推广。目前 中医临床中, 糖尿病的演变特点为阴虚热盛型、气阴两虚型、 阴阳两虚型 3 型, 阴虚作为 3 型之共性, 贯穿病之始终, 为病 之本; 热盛、湿浊、血瘀则为病之标, 对 2 型糖尿病患者证候的 分型, 应当清晰明确, 以尽可能提高治疗效果并改善预后。

\section{5 结语}

通过中医药对 2 型糖尿病的治疗是一个几长复杂的过 程, 其治疗的有效与否不能与西医降糖药的疗效相比较, 一 时血糖的高低不能成为中医论治 2 型糖尿病是否有效的标 准, 因此对于 2 型糖尿病的治疗, 应当根据患者疾病的不同阶 段、临床症状把握时机采取对应的治疗方式, 随机应变并持之 以恒。

综上所述, 对于 2 型糖尿病患者的治疗, 应用辨体-辨病辨证诊疗模式对其进行辨证诊治极具临床价值, 有较强的指 导性与可操作性, 此种辨证诊疗模式值得临床推广。

\section{参考文献}

[1]段利平.三位一体护理干预在老年 2 型糖尿病患者中的应用观 察 [J].首都食品与医药,2020,27(11):178.

[2]申子龙,赵文景, 蔡朕,等.从气、血、水“三位一体”论治糖尿病肾 脏病探析[J].现代中医药,2019,39(5):37-39.

[3]金玲玲, 叶青,马雨杨,等.社区 2 型糖尿病患者自我管理能力 “三位一体”糖尿病精细化管理模式干预效果评价[J].中国公共卫生, 2020,36(5):810-813.

[4]吴丹,洪普,王娟,等.多学科诊疗模式下 “三位一体”无缝隙护理 服务在妊娠期糖尿病患者中的应用[J].中国医药导报,2016,13(30):114-117. 